\title{
Miranda
}

Revue pluridisciplinaire du monde anglophone /

Multidisciplinary peer-reviewed journal on the English-

speaking world

Interview de Graham Kotowich, chorégraphe, et d'Aurélie Barré, assistante à la mise en scène dans Frankenstein Junior, comédie musicale d'après le film de Mel Brooks (1974), à l'affiche à l'OpéraThéâtre de Metz (octobre 2021)

\section{Nathalie Vincent-Arnaud}

\section{OpenEdition}

Édition électronique

URL : https://journals.openedition.org/miranda/40844

DOI : 10.4000/miranda.40844

ISSN : 2108-6559

Éditeur

Université Toulouse - Jean Jaurès

Référence électronique

Nathalie Vincent-Arnaud, « Interview de Graham Kotowich, chorégraphe, et d'Aurélie Barré, assistante à la mise en scène dans Frankenstein Junior, comédie musicale d'après le film de Mel Brooks (1974), à l'affiche à l'Opéra-Théâtre de Metz (octobre 2021) », Miranda [En ligne], 23 | 2021, mis en ligne le 04 octobre 2021, consulté le 29 novembre 2021. URL : http://journals.openedition.org/miranda/40844 ; DOI : https://doi.org/10.4000/miranda.40844

Ce document a été généré automatiquement le 29 novembre 2021.

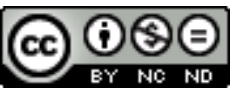

Miranda is licensed under a Creative Commons Attribution-NonCommercial-NoDerivatives 4.0 International License. 


\title{
Interview de Graham Kotowich, chorégraphe, et d'Aurélie Barré, assistante à la mise en scène dans Frankenstein Junior, comédie musicale d'après le film de Mel Brooks (1974), à l'affiche à l'Opéra- Théâtre de Metz (octobre 2021)
}

\author{
Nathalie Vincent-Arnaud
}

Nathalie Vincent-Arnaud: Pourriez-vous vous présenter en définissant vos rôles respectifs dans ce spectacle (créé à Broadway en 2007 et dont l'adaptation française a été jouée au théâtre Dejazet à Paris) ?

Aurélie Barré et Graham Kotowich : Aurélie Barré, assistante à la mise en scène sur ce spectacle, Frankenstein Junior, après avoir été danseuse à l'Opéra-Théâtre de Metz pendant 10 ans. Je suis le bras droit du metteur en scène, Paul-Émile Fourny, et j'assiste à toutes les séances de travail jusqu'à la première représentation. Mon rôle est de rédiger le cahier de régie, c'est-à-dire de noter tout ce qui se passe en répétition, d'assurer une bonne communication entre toutes les équipes, et d'agir à titre d'intermédiaire entre les différents intervenants(directeur de production, directeur technique et équipe de production).

Graham Kotowich, chorégraphe après avoir été danseur au Northern Ballet Company in Leeds puis à l'Opéra-Théâtre de Metz. Je suis chargé de la chorégraphie du spectacle.

NVA: L'œuvre était programmée l'an passé à l'Opéra-Théâtre de Metz mais les représentations ont dû être annulées en raison de la pandémie. Sa reprogrammation cette année se charge-t-elle pour vous d'une signification particulière parmi les autres spectacles 
proposés? Et ce délai a-t-il fait évoluer le regard que vous portez sur elle ou la préparation que vous avez effectuée?

$\mathrm{AB}$ et $\mathrm{GK}$ : Le travail réalisé l'année dernière avec tous les artistes a été remarquable et nous étions tous très enthousiastes à l'idée de faire découvrir cette production au public. Les reprogrammations des pièces ne laissent guère beaucoup plus de temps pour un travail de fond dans un planning très serré et contraint par les "bouchons" occasionnés dans le contexte de la pandémie. Mais nous étions prêts l'an dernier et le serons également cette année. Le temps qui s'est écoulé est précieux puisque chaque artiste aura eu l'opportunité de faire évoluer et mûrir son personnage.

NVA : Pour ce qui est de la chorégraphie, aviez-vous déjà une expérience dans le domaine de la comédie musicale? Et comment avez-vous abordé cette œuvre en particulier, en relation avec votre propre rôle de danseur?

GK : Mes expériences en matière de comédie musicale ont commencé tôt dans ma jeunesse. J'étais plutôt intéressé par la danse classique mais j'ai également participé à des spectacles comme Les Misérables. Professionnellement j'ai surtout travaillé dans des compagnies de danse classique mais en 2013/2014 j'ai participé à une production contemporaine, Swan Lake, de Matthew Bourne. La compagnie est réputée pour engager des danseurs avec des profils différents. Après cette expérience, j'ai repris des cours de chant et participé à une production, Once Upon a Mattress, et j'ai même osé une audition pour Un Américain à Paris! Après une série d'auditions, j'ai finalement intégré le ballet de l'Opéra-Théâtre de Metz Métropole. À Metz, j'ai eu la chance de participer à des productions variées: ballets classiques, pièces contemporaines, comédies musicales (telles L'Auberge du Cheval Blanc ou La Vie parisienne). Ensuite, le metteur en scène et directeur de l'Opéra-Théâtre, Paul-Emile Fourny, m'a confié la chorégraphie de Frankenstein Junior après avoir découvert mon travail autour d'un projet de cartes blanches avec le ballet, dans le cadre des journées du patrimoine. C'est une véritable chance d'être passé de l'autre côté du studio pour effectuer ce travail avec mes collègues dans le cadre d'une nouvelle aventure créative.

NVA : Trouve-t-on des chorus lines, des solos, des duos comme dans la chorégraphie d'une comédie musicale américaine classique ou plus récente à la Jerome Robbins, ou la forme est-elle un peu plus atypique?

GK : La chorégraphie de Frankenstein Junior est une combinaison de différentes idées mais dès le début j'avais envie d'y incorporer de la gestuelle de danse contemporaine. Les protagonistes ont plusieurs solos, des duos et participent aux danses d'ensemble. Leurs mouvements sont en accord avec le caractère de leur personnage et c'était très enrichissant de pouvoir en discuter avec eux. Les danses d'ensemble sont toutes très différentes mais sont surtout créées en fonction de la musique et de la narration. Certains numéros sont plus fidèles à la tradition de la comédie musicale avec notamment le "grand final" qui dégage une folle énergie. D’autres moments dansés répondent à des propositions plus abstraites. Durant le processus de création je recherchais comment interpréter la musique dans mon propre corps, et ensuite avec les corps de mes collègues danseurs.

NVA : Les danseurs de l'Opéra-Théâtre sont-ils impliqués au même titre que les chanteurs? Et surtout, comment s'organise le travail lorsqu'il faut, sur scène, réunir ces deux champs de compétence au sein des différents rôles?

$\mathrm{AB}$ et $\mathrm{GK}$ : Les danseurs ayant des rôles chantés dans l'œuvre ont des formations lyriques en plus de leurs métiers d'artistes chorégraphiques. Tous les danseurs ont 
travaillé les parties chantées en amont et les choristes ont quant à eux travaillé les parties dansées. Ce n'était pas évident d'organiser les répétitions dans le contexte restrictif de la pandémie mais à partir du moment où les artistes ont pu répéter tous ensemble sur le plateau c'est devenu plus facile. Les détails se mettent plus facilement en place sur scène et la production prend forme. Malheureusement nous n'avons pas pu le présenter au public l'année dernière mais nous en avons gardé une captation comme outil de travail, et nous sommes très heureux de le partager cette année dans cet univers si particulier de Mel Brooks.

NVA: La comédie musicale exerce-t-elle à votre avis une fascination particulière sur le public français contemporain, et pourquoi?

$A B$ et GK : Nous pensons que oui car elle réunit beaucoup d'artistes en scène, chose rare actuellement dans le contexte de pandémie avec des budgets resserrés au moment du choix des programmations. Elle reste fascinante car elle permet de découvrir des artistes aux talents multiples : chant, musique, danse. Les spectateurs sont embarqués dans la narration avec une curiosité enfantine et les numéros d'ensemble sont des moments exaltants qui donnent une énergie communicative. On ne peut que penser à la phrase de Mel Brooks: "If you're quiet, you're not living. You've got to be noisy and colorful and lively."

\section{BIBLIOGRAPHIE}

Liens utiles :

https://opera.eurometropolemetz.eu/fr/a-l-affiche/frankenstein-junior_-d.html

https://www.szenik.eu/fr/event/frankenstein-junior

\section{INDEX}

Mots-clés : Broadway, chorégraphie, comédie musicale, Mel Brooks, Opéra-Théâtre de Metz Keywords : Broadway, choreography, musical, Mel Brooks, Opéra-Théâtre de Metz

Thèmes : Dance

\section{AUTEURS}

\section{NATHALIE VINCENT-ARNAUD}

Professeur

Université Toulouse-Jean Jaurès

nathalie.vincent-arnaud@univ-tlse2.fr 